\title{
Molecular Basis of Macrolide Resistance in Campylobacter Strains Isolated from Poultry in South Korea
}

\author{
Bai Wei (i) and Min Kang (iD \\ Department of Veterinary Infectious Diseases and Avian Diseases, College of Veterinary Medicine and Center for Poultry \\ Diseases Control, Chonbuk National University, Jeonju, Republic of Korea
}

Correspondence should be addressed to Min Kang; vet.minkang@gmail.com

Received 11 March 2018; Accepted 19 June 2018; Published 5 July 2018

Academic Editor: María de Guía Córdoba

Copyright (c) 2018 Bai Wei and Min Kang. This is an open access article distributed under the Creative Commons Attribution License, which permits unrestricted use, distribution, and reproduction in any medium, provided the original work is properly cited.

\begin{abstract}
We investigated the molecular mechanisms underlying macrolide resistance in 38 strains of Campylobacter isolated from poultry. Twenty-seven strains were resistant to azithromycin and erythromycin, five showed intermediate azithromycin resistance and erythromycin susceptibility, and six showed azithromycin resistance and erythromycin susceptibility. Four Campylobacter jejuni and six Campylobacter coli strains had azithromycin MICs which were 8-16 and 2-8-fold greater than those of erythromycin, respectively. The A2075G mutation in the 23S rRNA gene was detected in 11 resistant strains with MICs ranging from 64 to $\geq$ $512 \mu \mathrm{g} / \mathrm{mL}$. Mutations including V137A, V137S, and a six-amino acid insertion (114-VAKKAP-115) in ribosomal protein L22 were detected in the $C$. jejuni strains. Erythromycin ribosome methylase B-erm(B) was not detected in any strain. All strains except three showed increased susceptibility to erythromycin with twofold to 256-fold MIC change in the presence of phenylalanine arginine $ß$-naphthylamide (PAßN); the effects of PAßN on azithromycin MICs were limited in comparison to those on erythromycin MICs, and 13 strains showed no azithromycin MIC change in the presence of PAßN. Differences between azithromycin and erythromycin resistance and macrolide resistance phenotypes and genotypes were observed even in highly resistant strains. Further studies are required to better understand macrolide resistance in Campylobacter.
\end{abstract}

\section{Introduction}

Infection with Campylobacter spp. is considered to be the most common cause of bacterial gastroenteritis in humans worldwide. Macrolides are considered the first drug of choice for treating Campylobacter gastroenteritis. Resistance to macrolides has been reported in a few scattered clinical isolates of Campylobacter across the world. High prevalence of macrolide-resistant Campylobacter spp., especially C. coli, in animal meat has been reported [1], and this finding is of concern because of the risk of transmission of such isolates to human.

Modification of the antibiotic target genes via methylation or mutation, and efflux of antibiotics from bacterial cells could induce macrolide resistance [2]. The most important macrolide resistance mechanism in Campylobacter involves the modification of ribosomal target sites and weakening of the interaction between the tunnel wall of the ribosome and the macrocyclic ring of the macrolide [3]. Point mutations in domain $\mathrm{V}$ of the $23 \mathrm{~S}$ rRNA at positions 2,074 and 2,075 are the most common mechanisms for high-level macrolide resistance in Campylobacter spp. [4]. Several modifications in the ribosomal proteins L4 and L22 are associated with lowto intermediate-level macrolide resistance in Campylobacter [4]. The chromosomally encoded multidrug resistancenodulation-cell division (RND) efflux system is involved in intrinsic and acquired macrolide resistance in Campylobacter spp. [4]. A ribosomal methylase, encoded by the erythromycin ribosome methylase B-erm(B) gene, located in the chromosomal multidrug resistance genomic island (MDRGI) in C. coli from swine, was reported for the first time in China in 2014 [5]. Subsequently, several reports emerged of erm(B)-harboring C. jejuni in animal meat including that of swine and chicken and in human diarrheal samples [6, 7]. Outside China, an erm (B)-positive C. coli strain was isolated from chicken in Spain in 2016 [8]. 
Macrolides are a class of natural or semisynthetic products comprising a large macrocyclic lactone ring to which one or more deoxy sugars are attached [2]. The lactone rings can be either 14-membered (clarithromycin, dirithromycin, erythromycin, and roxithromycin), 15membered (azithromycin), or 16-membered (josamycin, kitasamycin, spiramycin, and tylosin) [2]. In general, modification of ribosomal targets and drug efflux confer cross-resistance to macrolides. During antibiotic treatment in clinical settings, erythromycin and azithromycin are widely used because they have a broad spectrum of activity not only against Gram-positive bacteria, but also against Gram-negative bacteria [9]. Azithromycin is more potent than erythromycin against Gram-negative bacteria including Campylobacter, with a lower MIC [9]. However, azithromycin shows a higher MIC than erythromycin against Gram-positive bacteria and rarely acts against Gram-negative bacteria $[10,11]$. The diverse mechanisms underlying resistance to erythromycin and azithromycin continue to be unclear.

It is well known that the handling and/or consumption of chicken meat are the main causes of human infection with Campylobacter. Other poultry sources also pose a similar threat to human health. Even though the vertical transmission of Campylobacter is questionable, breeder chicken harboring antibiotic-resistant bacteria could be a public health threat as they can horizontally transmit antibiotic-resistant Campylobacter to broiler chicken in the production chain, indirectly leading to human infection [12]. With the increasing consumption of duck meat around the world and worldwide reports of duck-related productinduced human campylobacteriosis, researchers are focusing more on antibiotic-resistant Campylobacter found in duck $[13,14]$. Even though the occurrence of highly macrolideresistant Campylobacter in breeder chicken and macrolideresistant Campylobacter in duck meat has been reported $[15,16]$, the causes of macrolide resistance in these species have rarely been reported. Therefore, we investigated the genetic basis of macrolide resistance in Campylobacter from poultry sources including breeder chicken and chicken and duck meat and identified the isolated strains as C. jejuni and $C$. coli; these strains showed different levels of resistance to azithromycin and erythromycin, evaluated using molecular methods. Additionally, we sought to investigate the diverse mechanisms underlying the higher resistance to azithromycin, but not to erythromycin, shown by the Campylobacter strains.

\section{Materials and Methods}

2.1. Origin of Campylobacter Strains and Minimal Inhibitory Concentrations (MIC) Determination. A total of 38 strains of Campylobacter (15 strains of C. jejuni and 23 strains $C$. coli) showing either intermediate resistance or resistance to azithromycin and erythromycin were used in this study (Tables 1 and 2). The strains were isolated from poultry between 2013 and 2016 in a previous study [16]. The sample sources were divided into four types, and the number of strains collected from each source was as follows: (1) in 176 feces samples from breeder chicken farms, 88 isolates of Campylobacter were collected and 17 strains (one C. jejuni and $16 \mathrm{C}$. coli) showed resistance to azithromycin; (2) in 1,003 samples (feces and environmental samples) from broiler chicken farms, 55 isolates were collected and none of them showed resistance to azithromycin or erythromycin; (3) in 249 chicken meat samples from retail markets, 104 isolates were collected and 15 strains (10 C. jejuni and five C. coli) showed resistance to azithromycin; and (4) in 106 duck meat sample from retail markets, 102 isolates were collected and six strains (four C. jejuni and two C. coli) showed resistance to azithromycin. The MICs of azithromycin and erythromycin were determined using agar or broth dilution methods and the breakpoints were as defined by the National Antimicrobial Resistance Monitoring System (NARMS) for azithromycin: susceptible, $\leq 2 \mu \mathrm{g} / \mathrm{mL}$; intermediate, $4 \mu \mathrm{g} / \mathrm{mL}$; and resistant, $\geq 8 \mu \mathrm{g} / \mathrm{mL}$. The MIC breakpoints for erythromycin were susceptible, $\leq 8 \mu \mathrm{g} / \mathrm{mL}$; intermediate, 16 $\mu \mathrm{g} / \mathrm{mL}$; and resistant, $\geq 32 \mu \mathrm{g} / \mathrm{mL}$ [17]. The reference strain C. jejuni ATCC 33560 was used as the quality control strain.

2.2. Characterization of Macrolide Resistance in Campylobacter Strains. Genomic DNA templates for PCR were prepared using fresh Campylobacter colonies on $5 \%$ sheep blood agar plates (Komed, Seongnam, South Korea) by adding $100 \mu \mathrm{l}$ sterile distilled water and boiling in a heater block at $100^{\circ} \mathrm{C}$ for $15 \mathrm{~min}$. Mutations at positions 2,074 and 2,075 of the domain $\mathrm{V}$ of $23 \mathrm{~S}$ rRNA gene were analyzed by sequencing all three copies of $23 \mathrm{~S}$ rRNA. Three separate reactions were employed to amplify the three copies of the 23S rRNA gene in all C. jejuni and C. coli strains [3]. Subsequently, potential macrolide resistance-associated mutations were identified by sequencing a 308-bp fragment from each copy of the target gene [3].

In addition, to assess the contribution of mutations within the ribosomal genes $r p l D$ and $r p l V$ encoding L4 and L22, respectively, to macrolide resistance, sequence analysis of these genes was performed for all 38 strains. L4- and L22-encoding genes were amplified as previously described [18]. The presence of the recently reported macrolide resistance-related ribosomal RNA methylase gene, erm(B), was confirmed using a method described by Zhang et al. [6]. To investigate the role of drug efflux in macrolide resistance, $c m e B$, an efflux pump gene (1,070 bp), was amplified using the method described by Pumbwe et al. [19].

PCR products were purified using JET-SORB gel extraction kit (Genomed, Kampenhout, Belgium) following the manufacturer's instructions. Subsequently, they were sequenced using an ABI 3100 Genetic Analyzer (Applied Biosystems, Foster City, CA). The sequences were analyzed and compared with the reference sequence using the software MEGA (version 5.0). A macrolide-susceptible strain of NCTC 11168 (GenBank: AL111168.1) was used as a reference strain to analyze the mutations in genes encoding $23 \mathrm{~S}$ rRNA, L4, and L22. 


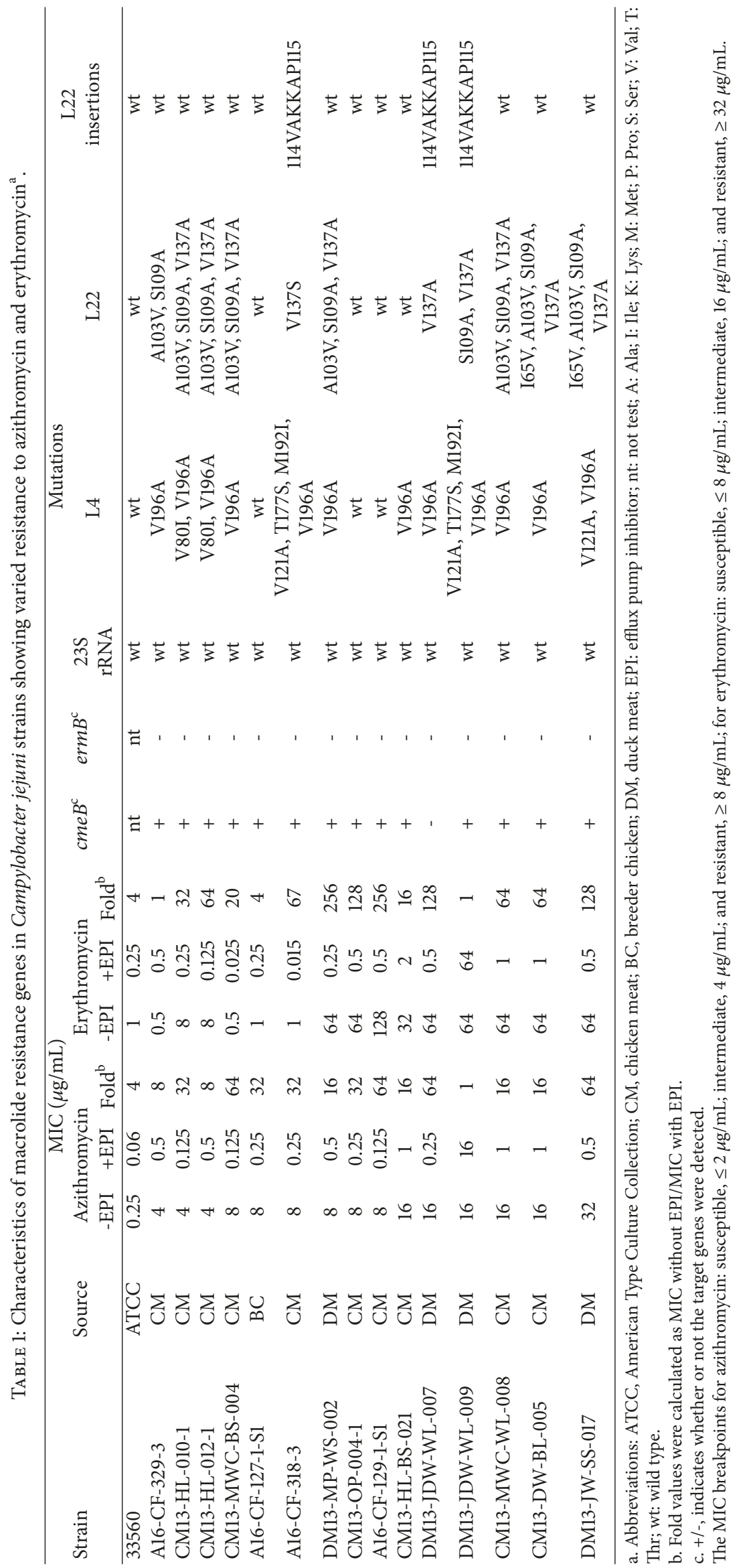




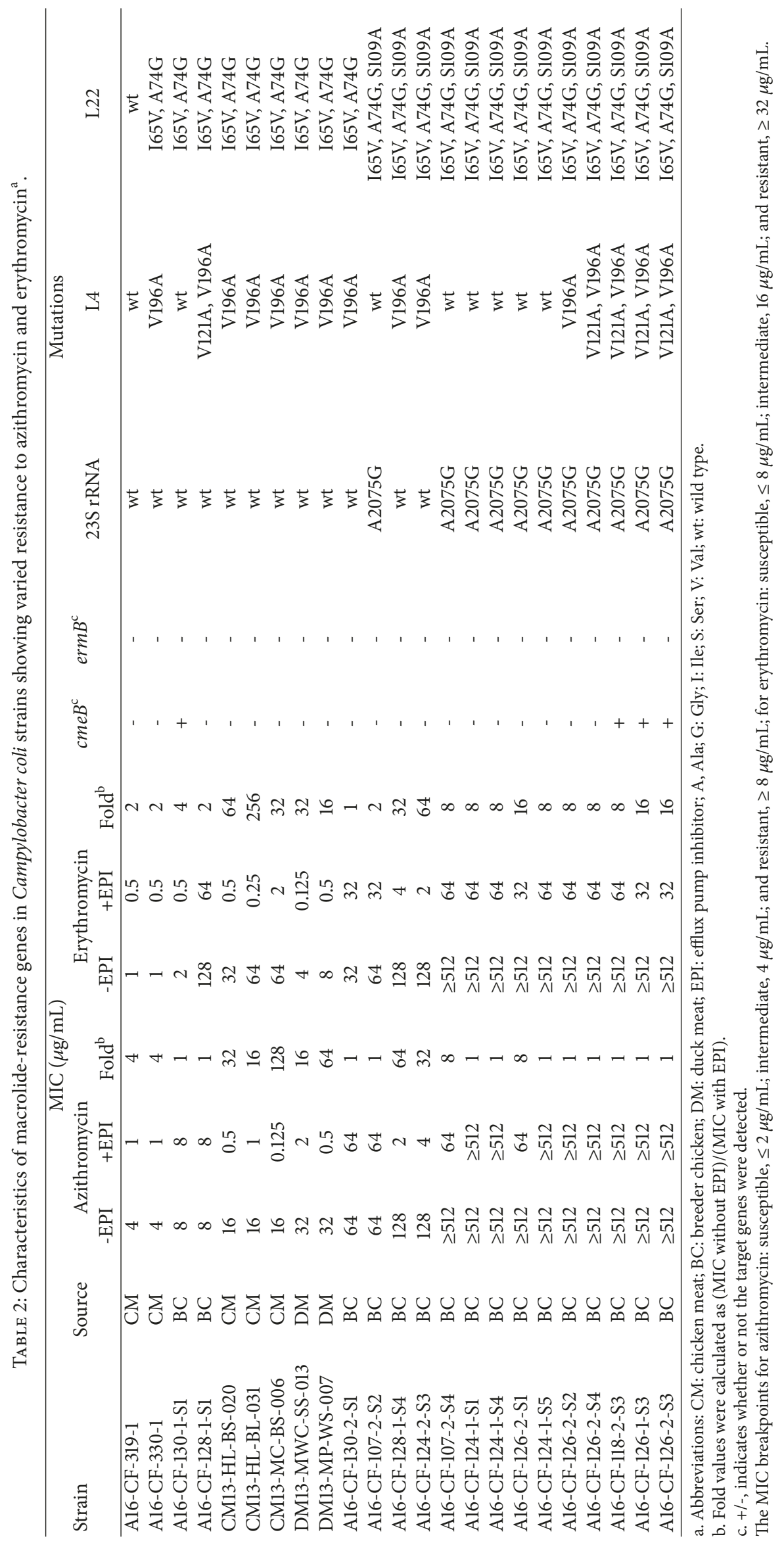


2.3. Effects of an Efflux Pump Inhibitor (EPI) on Macrolide Resistance. To investigate the contributions of efflux pump activity to macrolide resistance, the MICs of azithromycin and erythromycin were determined in presence of the EPI phenylalanine arginine $ß$-naphthylamide (PAßN, Sigma, St. Louis, Missouri). The broth microdilution method was used to determine the MICs in the presence of $20 \mu \mathrm{g} / \mathrm{mL}$ PAßN in Mueller-Hinton broth (Oxoid, Basingstoke, England). The $C$. jejuni ATCC 33560 was used as the reference strain.

\section{Results}

3.1. Variation in Resistance to Azithromycin and Erythromycin. Among the 38 tested Campylobacter strains, 27 strains, of which nine were $C$. jejuni and 18 were $C$. coli, were resistant to azithromycin and erythromycin (Tables 1 and 2). A total of five strains (three C. jejuni and two C. coli) showed intermediate resistance to azithromycin with an MIC of 4 $\mu \mathrm{g} / \mathrm{mL}$ and were susceptible to erythromycin with MICs ranging from 0.5 to $8 \mu \mathrm{g} / \mathrm{mL}$. A total of six strains (three $C$. jejuni and three C. coli) were resistant to azithromycin and susceptible to erythromycin with MICs ranging from 8-32 $\mu \mathrm{g} / \mathrm{mL}$ and $0.5-8 \mu \mathrm{g} / \mathrm{mL}$, respectively. MICs of azithromycin against four strains of C. jejuni (A16-CF-329-3, CM13-MWCBS-004, A16-CF-127-1-S1, and A16-CF-318-3) and six strains of C. coli (A16-CF-319-1, A16-CF-330-1, A16-CF-130-1-S1, DM13-MWC-SS-013, DM13-MP-WS-007, and A16-CF-130-2S1) were 8-16 and 2-8-fold higher, respectively, than those of erythromycin.

3.2. Sequence Analysis of the $23 S$ rRNA. All of the 15 C. jejuni strains, including one strain (A16-CF-129-1-S1) showing a high-level resistance to erythromycin with an MIC of 128 $\mu \mathrm{g} / \mathrm{mL}$, harbored a wild-type $23 \mathrm{~S}$ rRNA sequence (Table 1 ).

The point mutation A2075G was detected in all three copies of the 23S rRNA gene from 11 strains of C. coli, which were resistant to azithromycin and erythromycin with MICs ranging from 64 to $\geq 512 \mu \mathrm{g} / \mathrm{mL}$ (Table 2). Two strains of C. coli (A16-CF-128-1-S4 and A16-CF-124-2-S3) showed highlevel resistance to azithromycin and erythromycin (MIC = $128 \mu \mathrm{g} / \mathrm{mL})$ and did not harbor any mutations in the $23 \mathrm{~S}$ rRNA gene, and one strain (A16-CF-128-1-S1) showed highlevel resistance to erythromycin $(\mathrm{MIC}=128 \mu \mathrm{g} / \mathrm{mL})$ and did not harbor any mutations in the $23 \mathrm{~S}$ rRNA gene. The other five strains of C. coli were resistant to both azithromycin and erythromycin, with no mutations in the $23 \mathrm{~S}$ rRNA gene.

3.3. Investigation of the Ribosomal Proteins L4 and L22. Analysis of amino acid sequences of the ribosomal proteins L22 and L4 from the C. jejuni and C. coli strains revealed the presence of different combinations of amino acid substitutions (Tables 1 and 2). The following amino acid substitutions were identified in L4 from C. jejuni; V80I, T177S, and M192I in two isolates, V121A in three isolates, and V196A in 12 isolates. The following amino acid substitutions were identified in $\mathrm{L} 22$ from $C$. jejuni; $\mathrm{I} 65 \mathrm{~V}$ in two isolates, A103V in eight isolates, S109A in nine isolates, V137S in one isolate, and V137A in eight isolates. In addition to the amino acid substitutions observed in L22 sequences, a six-amino acid sequence (VAKKAP) present between positions 114 and 115 was also identified in three azithromycin-resistant strains of $C$. jejuni.

Minimal genetic diversity in L4 and L22 amino acid substitutions was observed in 23 strains of $C$. coli from poultry. The substitution V121A was identified in L4 from five C. coli strains and the substitution V196A was identified in $\mathrm{L} 4$ from $15 \mathrm{C}$. coli strains; the substitutions $\mathrm{I} 65 \mathrm{~V}$ and A74G were identified in L22 from nine C. coli strains and the substitutions I65V, A74G, and S109A were identified in L22 from 13 C. coli strains.

3.4. PCR Detection of the cmeB and erm(B) Gene. The presence of cmeB in 14 (93.3\%) C. jejuni strains and 4 (17.4 \%) C. coli strains was confirmed using PCR. However, none of the investigated strains of $C$. jejuni and C. coli harbored $\operatorname{erm}(\mathrm{B})$, as observed in the PCR results obtained.

3.5. Efficacy of an EPI. The effects of PAßN on the MICs of macrolide antibiotics in the C. jejuni and C. coli strains are shown in Tables 1 and 2. The presence of PAßN greatly decreased the MICs of azithromycin and erythromycin against most of $C$. jejuni and C. coli strains. In the Campylobacter strains with no mutations in the 23S rRNA gene, all the azithromycin intermediate/resistant strains were restored to susceptibility except for four strains (DM13-JDW-WL009, A16-CF-128-1-S1, A16-CF-130-2-S1, and A16-CF-130-1S1), and all erythromycin-resistant strains were restored to susceptibility except three strains (DM13-JDW-WL-009, A16CF-128-1-S1, and A16-CF-130-2-S1).

All the strains showed increased susceptibility to erythromycin in the presence of PAßN with at least two-fold to 256-fold MIC change, except two $C$. jejuni strains and one $C$. coli strain which showed no MIC change. The effect of PAßN on azithromycin MIC was lesser than that on erythromycin MIC, with 13 strains (one C. jejuni and 12 C. coli) of the 38 strains showing no MIC change in the presence of PAßN. In addition, of the 11 strains carrying mutations in the $23 \mathrm{~S}$ rRNA gene, nine showed no change in azithromycin MIC while all 11 strains showed a 2-16-fold decrease in erythromycin MIC.

\section{Discussion}

In this study, Campylobacter strains isolated from breeder chicken and chicken and duck meat between 2013 and 2016 were used to investigate the molecular mechanisms underlying macrolide resistance in C. jejuni and C. coli. Our data revealed that point mutations at positions 2,075 in domain $\mathrm{V}$ of the $23 \mathrm{~S}$ rRNA gene contributed to high-level azithromycin and erythromycin resistance in 11 Campylobacter strains. These mutations were not present in C. jejuni and C. coli strains with a low-level or intermediate resistance to azithromycin and erythromycin. This finding supports previously published reports that suggested a predominant role for this mutation in macrolide resistance $[4,20]$. The binding site substitutions of A2075G, A2074G, and A2074C in the $23 \mathrm{~S}$ rRNA gene in C. jejuni and C. coli have been implicated 
in high-level resistance to azithromycin and erythromycin in the field and in the laboratory [4]. The substitution A2075G was the most prevalent genetic mutation conferring high macrolide resistance in the field, suggesting A2075G may provide specific biological or survival advantages compared to $\mathrm{A} 2074 \mathrm{G}$ and $\mathrm{A} 2074 \mathrm{C}[3,20-22]$. Our results were also consistent with those of previous studies in Korea that suggested that the A2075G mutation in the 23S rRNA gene appeared to be the main contributor to high macrolide resistance $[23,24]$.

As observed in several other species of Gram-negative bacteria, RND efflux pumps confer resistance to macrolides in Campylobacter spp. Inhibition of the efflux pumps using EPIs increases the susceptibility of Campylobacter to macrolides [20]. Our results showed that an EPI promoted a marked decrease in resistance to both azithromycin and erythromycin in most of Campylobacter strains (Tables 1 and 2). In highly erythromycin-resistant strains, the presence of the A2075G 23S rRNA gene mutation and efflux pump activity indicated synergism between these two resistance mechanisms in Campylobacter [20]. However, this may not apply to azithromycin because nine out of 11 strains with the A2075G 23S rRNA mutation showed no azithromycin MIC change in the presence of PAßN. Our result was consistent with that of a previous study which found that high azithromycin resistance in Campylobacter was mainly due to the A2075G 23S rRNA mutation [25]. These results suggest that the A2075G 23S rRNA mutation in Campylobacter was sufficient to confer high-level resistance to azithromycin, while the mutation synergizes with the drug efflux pump system to confer high erythromycin resistance. Further studies are required to assess the contribution of different mechanisms to erythromycin and azithromycin resistance in Campylobacter.

A number of previous studies have reported that modifications in the ribosomal proteins L4 and L22 were associated with a lower level of macrolide resistance [26]. Numerous substitutions and insertions in the ribosomal protein sequences in macrolide-resistant strains have been documented. Amino acids at the positions 63-74 are a part of the most important target region in L4; no variation was found in this region of L4 in the present study. In contrast, the most frequent changes, V121A and V196A, were located outside the important target region. The mutations at positions 121 and 196 were identified in susceptible and resistant isolates in previous studies. This suggests that these substitutions are unlikely to contribute directly to macrolide resistance [27]. Other substitutions such as V80I, T177S, and M192I were also identified in erythromycin-susceptible and -resistant isolates previously [23, 27]. In L22, substitutions including I65V, A74G, A103V, and S109A were identified in erythromycin-susceptible and -resistant isolates previously $[27,28]$. The mutations V137A and V137S, located in the $\beta$ hairpin region close to the $\mathrm{C}$-terminus, were identified in C. jejuni strains. L22 consists of a small $\alpha$ plus $\beta$ domain, with the $\beta$-hairpin contributing to the formation of the polypeptide tunnel exit at the surface of the ribosome. The mutation in this domain might change the surface properties and block macrolide binding [29]. Nevertheless, isolation of Campylobacter strains harboring such substitutions and showing reduced susceptibility to erythromycin did not lead to the elucidation of the mechanistic significance of these amino acid substitutions in the present study.

In addition to single amino acid substitutions, a sixamino acid insertion (114-VAKKAP-115) within the $\beta$-hairpin region of L22 was found in three $C$. jejuni strains showing azithromycin resistance and reduced susceptibility to erythromycin (Table 1). Amino acid insertions in L22 have been reported in a number of bacterial species, both Gramnegative and Gram-positive [30, 31]. For example, insertions at position 86 or 98 in L22 reportedly conferred macrolide resistance in C. jejuni and C. coli [26], and a six-amino acid insertion between T108 and V109 in L22 of Streptococcus conferred resistance to azithromycin and erythromycin [32]. The significance of this insertion in L22 of Campylobacter in relation to macrolide resistance needs to be investigated. In the present study, all strains which showed reduced susceptibility to azithromycin and erythromycin, except one C. coli strain, carried the mutations I65V and A74G. These substitutions were not observed in C. jejuni strains. The significance of the coexistence of these amino acid substitutions is unknown. The occurrence of such mutations may be associated with local environmental conditions and other selective pressures; most of the previously reported $C$. coli strains, carrying both the amino acid substitutions, were isolated from chicken and swine in Korea [23]. Further studies are required to assess whether the coexistence of these amino acid substitutions contributes to Campylobacter macrolide resistance.

Erythromycin, the first 14-membered macrolide, is active against Gram-positive and some Gram-negative microorganisms. To improve acid stability and oral bioavailability of erythromycin, the first 15-membered macrolide, azithromycin, was developed by inserting a basic nitrogen atom into the macrocyclic ring [33]. Azithromycin exhibited enhanced in vitro and in vivo potency against Gram-positive and Gramnegative bacteria compared to erythromycin [34]. Bacteriostatic and bactericidal activity of azithromycin against Campylobacter was up to four times more potent than that of erythromycin [9]. In this study, we found four C. jejuni strains and six C. coli strains showing higher resistance to azithromycin than to erythromycin; and the MIC of azithromycin was 8-16 and 2-8-fold higher against the $C$. jejuni strains and the $C$. coli strain, respectively, compared to that of erythromycin. Additionally, even in the presence of PAßN, two C. jejuni strains (CM13-MWC-BS004 and A16-CF-318-3) and five C. coli (A16-CF-319-1, A16CF-330-1, A16-CF-130-1-S1, DM13-MWC-SS-013, and A16CF-130-2-S1) showed higher MICs of azithromycin than of erythromycin. These results were in agreement with those of previous studies in which erythromycin had a lower MIC than azithromycin against both Campylobacter and other Gram-positive bacteria [10, 23, 35, 36]. Ribosomal protein polymorphisms might affect the MICs of different macrolides, and an amino acid substitution (A86E) was identified in L22 from azithromycin-resistant and erythromycinsusceptible Campylobacter in a previous study [37]. Further, azithromycin was found to be less affected by drug efflux compared with erythromycin, and inactivation of the $c m e B$ 
gene led to greater MIC change of erythromycin than of azithromycin [25]. In the present study, the substitution A86E was not observed in L22 from C. jejuni and C. coli strains, and higher MICs of azithromycin than of erythromycin were observed even in the presence of an EPI. This may indicate different contributing factors for macrolide resistance, apart from efflux pump-mediated mechanisms. Further studies are required to verify the diverse mechanisms underlying erythromycin and azithromycin resistance and to facilitate the development of strategies to control macrolide-resistant Campylobacter. In addition, considering that poultry meat is a common source of human pathogens, careful thought must be given to select effective antibiotics against Campylobacter strains from poultry meat showing higher resistance to azithromycin than to erythromycin.

A relatively low prevalence of $c m e B$ in $C$. coli strains compared with that in $C$. jejuni strains was found in this study. This was consistent with findings of a recent study on turkeys reported by Olah et al. [38]. The primers used were unable to detect $c m e B$ due to the high sequence variation of $c m e B$ in C. coli isolates [39]. Differences between C. jejuni and C. coli strains should be further investigated.

The gene $\mathrm{erm}(\mathrm{B})$ is involved in a major mechanism underlying macrolide resistance in other bacteria, especially Grampositive bacteria [2]. The transferable $\operatorname{erm}(\mathrm{B})$ gene located in MDRGI on the chromosome and plasmids with high prevalence in Gram-positive bacteria has been described in highly erythromycin-resistant Campylobacter strains [5]. An increase in the incidence of erm(B)-carrying Campylobacter strains was reported in China, and their prevalence increased from $0.7 \%$ during 2007-2009 to $6.4 \%$ during 2011-2012 [40]. It is noteworthy that a recent study showed a particularly high prevalence $(15.1 \%)$ of $\mathrm{erm}(\mathrm{B})$ in strains in a province of southern China in 2017 [41]. The isolation of an erm(B)carrying clinical C. jejuni strain in 1994 suggests that erm(B) has a property of diffusional spread along time and space [7]. Following the characterization of erm(B)-positive C. coli isolated from chicken in Europe, researchers focused on the transferable erm(B), located in MDRGI on the chromosome as well as on plasmids. The erm (B) gene has not been detected in macrolide-resistant $C$. jejuni and $C$. coli from various sources including human clinical specimens, retail meat, and fecal samples from food animals in the USA or in macrolideresistant $C$. coli from colon contents of swine in France [37, 42]; in this study, erm(B) was not detected in C. jejuni and $C$. coli strains from poultry including breeder chicken or chicken and duck meat between 2013 and 2016. Continuous monitoring of erm(B) in Campylobacter is required, due to its highly transmittable nature.

Recent studies have shown that whole-genome sequencing (WGS) analysis can potentially be a rapid approach to define resistance genotypes and predict resistance phenotypes of bacteria with great sensitivity and specificity, and numerous proof-of-principle studies have also highlighted the value of WGS as a primary diagnostic tool to detect antibiotic resistance [43]. In Campylobacter, although a recent study showed that the correlation between resistance phenotypes and genotypes was $100 \%$ in terms of resistance to tetracycline, fluoroquinolones, and erythromycin
[37], numerous studies have reported differences between macrolide resistance phenotypes and genotypes of Campylobacter from various sources. These studies revealed that $C$. jejuni and C. coli strains were resistant to macrolides but did not harbor the corresponding genes or mutations required for resistance $[3,27,35,44]$. In this study, 27 Campylobacter strains with both azithromycin and erythromycin resistance did not harbor any mutations within the corresponding genes. Further, three strains were did not harbor any mutation even in the presence of EPI. In such a situation, even after WGS, phenotypic testing would be necessary to confirm macrolide susceptibility of strains given that only deep WGS can detect resistance.

In the present study, we investigated the macrolide resistance of $C$. jejuni and $C$. coli from breeder chicken and chicken and duck meat. Chicken meat is a well-known and a major source for campylobacteriosis in humans; macrolideresistant Campylobacter could be transmitted from duck and breeder chicken to humans. In our study, Campylobacter with high resistance to azithromycin and erythromycin was found in breeder chicken. Vertical transmission of Campylobacter is questionable and it has been reported that antibioticresistant Campylobacter from breeder chicken showed clonal homology to that found in humans [1]. In addition, the increased consumption of ducks, especially in Asia, also increases the risk of transmission of antibiotic-resistant Campylobacter to humans [13, 14]. Therefore, monitoring populations of macrolide-resistant Campylobacter in poultry, including breeder chicken and duck, is required.

It is noteworthy that all Campylobacter strains in the present study showing high resistance to azithromycin and erythromycin belonged to a single C. coli population isolated from breeder chicken. Similar to a previous study, macrolide resistance has been usually observed in C. coli, and it is believed that the high resistance in Campylobacter is due to extensive exposure to macrolide derivatives [45]. All Campylobacter strains with low or high macrolide resistance were found in breeder chicken from a single integrated company with no difference in management practices or antibiotic usage. This may suggest that polymorphisms, which cause macrolide resistance, could develop even under similar environment pressure. This is in agreement with a previous study in which it was shown that Campylobacter utilizes complex and different mechanisms to develop macrolide resistance in the field [46]. Therefore, further studies are required to elucidate mechanisms underlying the development of macrolide resistance in Campylobacter during chicken growth.

\section{Conclusions}

In conclusion, the data presented here confirmed previous findings, revealing that a mutation in the $23 \mathrm{~S}$ rRNA gene at position 2075 showed high-level azithromycin resistance in Campylobacter and that the 23S rRNA gene mutation acts synergistically with drug efflux to causes erythromycin resistance. Studies using a larger number of C. jejuni and C. coli strains showing high resistance to azithromycin but not erythromycin are required to investigate the diverse 
mechanisms underlying azithromycin and erythromycin resistance in Campylobacter spp. Further investigations are required to elucidate the significance of the observed amino acid substitutions V137A and V137S and the six-amino acid insertion identified in L22 from C. jejuni strains. None of the investigated strains of C. jejuni and C. coli from chicken and duck harbored erm(B); further surveillance might be required to confirm this. Because differences between macrolide resistance phenotypes and genotypes of Campylobacter showing high resistance were found, further studies are needed to improve our understanding of macrolide resistance in Campylobacter. To prevent and control macrolide resistance in Campylobacter, mechanisms underlying resistance development during chicken growth need to be elucidated. In addition, populations of macrolideresistant Campylobacter in poultry including breeder chicken and duck need to be monitored.

\section{Data Availability}

Sequence data that support the findings of this study have been deposited in the GenBank with the accession number MH084527-MH084640.

\section{Conflicts of Interest}

The authors have no conflicts of interest to declare.

\section{Acknowledgments}

This work was supported by Korea Institute of Planning and Evaluation for Technology in Food, Agriculture, Forestry, and Fisheries (nos. 716002-7, 315035-5), Basic Science Research Program through the NRF funded by the Ministry of Education (2017R1D1A1B03030883), and Research of Animal and Plant Quarantine Agency (Z-1543073-2015-16-01), South Korea.

\section{References}

[1] European Food Safety Authority, “The European Union summary report on trends and sources of zoonoses, zoonotic agents and food-borne outbreaks in 2011 has been published," Eurosurveillance, vol. 18, no. 15, pp. 45-45, 2013.

[2] M. Gaynor and A. S. Mankin, "Macrolide antibiotics: binding site, mechanism of action, resistance," Current Topics in Medicinal Chemistry, vol. 3, no. 9, pp. 949-960, 2003.

[3] A. Gibreel, V. N. Kos, M. Keelan et al., "Macrolide resistance in Campylobacter jejuni and Campylobacter coli: Molecular mechanism and stability of the resistance phenotype," Antimicrobial Agents and Chemotherapy, vol. 49, no. 7, pp. 2753-2759, 2005.

[4] T. Luangtongkum, B. Jeon, J. Han, P. Plummer, C. M. Logue, and Q. Zhang, "Antibiotic resistance in Campylobacter: Emergence, transmission and persistence," Future Microbiology, vol. 4, no. 2, pp. 189-200, 2009.

[5] S. Qin, Y. Wang, Q. Zhang et al., "Report of ribosomal RNA methylase gene erm(B) in multidrug-resistant Campylobacter coli," Journal of Antimicrobial Chemotherapy, vol. 69, no. 4, pp. 964-968, 2014.
[6] A. Zhang, L. Song, H. Liang et al., "Molecular subtyping and erythromycin resistance of Campylobacter in China," Journal of Applied Microbiology, vol. 121, no. 1, pp. 287-293, 2016.

[7] J. Zhou, M. Zhang, W. Yang, Y. Fang, G. Wang, and F. Hou, "A seventeen-year observation of the antimicrobial susceptibility of clinical Campylobacter jejuni and the molecular mechanisms of erythromycin-resistant isolates in Beijing, China," International Journal of Infectious Diseases, vol. 42, pp. 28-33, 2016.

[8] D. Florez-Cuadrado, M. Ugarte-Ruiz, A. Quesada, G. Palomo, L. Domínguez, and M. C. Porrero, "Description of an erm(B)carrying Campylobacter coli isolate in Europe," Journal of Antimicrobial Chemotherapy, vol. 71, no. 3, pp. 841-843, 2016.

[9] J. Retsema, A. Girard, W. Schelkly et al., "Spectrum and mode of action of azithromycin (CP-62,993), a new 15-memberedring macrolide with improved potency against gram-negative organisms," Antimicrobial Agents and Chemotherapy, vol. 31, no. 12, pp. 1939-1947, 1987.

[10] A. Canu, B. Malbruny, M. Coquemont, T. A. Davies, P. C. Appelbaum, and R. Leclercq, "Diversity of ribosomal mutations conferring resistance to macrolides, clindamycin, streptogramin, and telithromycin in Streptococcus pneumoniae," Antimicrobial Agents and Chemotherapy, vol. 46, no. 1, pp. 125-131, 2002.

[11] E. Giovanetti, M. P. Montanari, M. Mingoia, and P. E. Varaldo, "Phenotypes and genotypes of erythromycin-resistant Streptococcus pyogenes strains in Italy and heterogeneity of inducibly resistant strains," Antimicrobial Agents and Chemotherapy, vol. 43, no. 8, pp. 1935-1940, 1999.

[12] N. A. Cox, L. J. Richardson, J. J. Maurer et al., "Evidence for horizontal and vertical transmission in Campylobacter passage from hen to her progeny," Journal of Food Protection, vol. 75, no. 10, pp. 1896-1902, 2012.

[13] M. Abid, H. Wimalarathna, J. Mills et al., "Duck liver-associated outbreak of Campylobacter infection among humans, United Kingdom, 2011," Emerging Infectious Diseases, vol. 19, no. 8, pp. 1310-1313, 2013.

[14] F. Adzitey, N. Huda, and G. R. Rahmat Ali, "Prevalence and antibiotic resistance of Campylobacter, Salmonella, and $L$. monocytogenes in ducks: a review," Foodborne Pathogens and Disease, vol. 9, no. 6, pp. 498-505, 2012.

[15] D. Pérez-Boto, F. J. García-Peña, J. C. Abad-Moreno, and M. A. Echeita, "Antimicrobial susceptibilities of Campylobacter jejuni and Campylobacter coli strains isolated from two early stages of poultry production," Microbial Drug Resistance, vol. 19, no. 4, pp. 323-330, 2013.

[16] B. Wei, S.-Y. Cha, R.-H. Yoon et al., "Prevalence and antimicrobial resistance of Campylobacter spp. isolated from retail chicken and duck meat in South Korea," Food Control, vol. 62, pp. 63-68, 2016.

[17] National Antimicrobial Resistance Monitoring System, NARMS retail meat annual report, U.S. Food and Drug Administration, Washington, DC, USA, 2011.

[18] H. Hao, Z. Yuan, Z. Shen et al., "Mutational and transcriptomic changes involved in the development of macrolide resistance in Campylobacter jejuni," Antimicrobial Agents and Chemotherapy, vol. 57, no. 3, pp. 1369-1378, 2013.

[19] L. Pumbwe and L. J. V. Piddock, "Identification and molecular characterisation of $\mathrm{CmeB}$, a Campylobacter jejuni multidrug efflux pump," FEMS Microbiology Letters, vol. 206, no. 2, pp. 185-189, 2002. 
[20] H. Bolinger and S. Kathariou, "The current state of macrolide resistance in Campylobacter spp.: Trends and impacts of resistance mechanisms," Applied and Environmental Microbiology, vol. 83, no. 12, 2017.

[21] D. Pérez-Boto, J. A. López-Portolés, C. Simón, S. Valdezate, and M. A. Echeita, "Study of the molecular mechanisms involved in high-level macrolide resistance of Spanish Campylobacter jejuni and Campylobacter coli strains," Journal of Antimicrobial Chemotherapy, vol. 65, no. 10, pp. 2083-2088, 2010.

[22] A. Gibreel and D. E. Taylor, "Macrolide resistance in Campylobacter jejuni and Campylobacter coli," Journal of Antimicrobial Chemotherapy, vol. 58, no. 2, pp. 243-255, 2006.

[23] S.-K. Lim, D.-C. Moon, M. H. Chae et al., "Macrolide resistance mechanisms and virulence factors in erythromycin-resistant Campylobacter species isolated from chicken and swine feces and carcasses," Journal of Veterinary Medical Science, vol. 78, no. 12, pp. 1791-1795, 2016.

[24] E. Shin and Y. Lee, "Characterization of erythromycin-resistant porcine isolates of Campylobacter coli," Microbial Drug Resistance, vol. 16, no. 3, pp. 231-239, 2010.

[25] C. Cagliero, C. Mouline, S. Payot, and A. Cloeckaert, "Involvement of the CmeABC efflux pump in the macrolide resistance of Campylobacter coli," Journal of Antimicrobial Chemotherapy, vol. 56, no. 5, pp. 948-950, 2005.

[26] C. Cagliero, C. Mouline, A. Cloeckaert, and S. Payot, "Synergy between efflux pump $\mathrm{CmeABC}$ and modifications in ribosomal proteins L4 and L22 in conferring macrolide resistance in Campylobacter jejuni and Campylobacter coli," Antimicrobial Agents and Chemotherapy, vol. 50, no. 11, pp. 3893-3896, 2006.

[27] M. Lehtopolku, P. Kotilainen, M. Haanperä-Heikkinen et al., "Ribosomal mutations as the main cause of macrolide resistance in Campylobacter jejuni and Campylobacter coli," Antimicrobial Agents and Chemotherapy, vol. 55, no. 12, pp. 5939-5941, 2011.

[28] D. Corcoran, T. Quinn, L. Cotter, and S. Fanning, "An investigation of the molecular mechanisms contributing to highlevel erythromycin resistance in Campylobacter," International Journal of Antimicrobial Agents, vol. 27, no. 1, pp. 40-45, 2006.

[29] S. T. Gregory and A. E. Dahlberg, "Erythromycin resistance mutations in ribosomal proteins L22 and L4 perturb the higher order structure of $23 \mathrm{~S}$ ribosomal RNA," Journal of Molecular Biology, vol. 289, no. 4, pp. 827-834, 1999.

[30] M. Peric, B. Bozdogan, M. R. Jacobs, and P. C. Appelbaum, "Effects of an efflux mechanism and ribosomal mutations on macrolide susceptibility of Haemophilus influenzae clinical isolates," Antimicrobial Agents and Chemotherapy, vol. 47, no. 3, pp. 1017-1022, 2003.

[31] C. Fyfe, T. H. Grossman, K. Kerstein, and J. Sutcliffe, "Resistance to macrolide antibiotics in public health pathogens," Cold Spring Harbor Perspectives in Medicine, vol. 6, no. 10, 2016.

[32] E. Pérez-Trallero, J. M. Marimon, L. Iglesias, and J. Larruskain, "Fluoroquinolone and macrolide treatment failure in pneumococcal pneumonia and selection of multidrug-resistant isolates," Emerging Infectious Diseases, vol. 9, no. 9, pp. 1159-1162, 2003.

[33] D. Jelić and R. Antolović, "From Erythromycin to Azithromycin and New Potential Ribosome-Binding Antimicrobials," Antibiotics-Basel, vol. 5, no. 3, 2016.

[34] J. G. Den Hollander, J. D. Knudsen, J. W. Mouton et al., "Comparison of pharmacodynamics of azithromycin and erythromycin in vitro and in vivo," Antimicrobial Agents and Chemotherapy, vol. 42, no. 2, pp. 377-382, 1998.
[35] S. R. Ladely, R. J. Meinersmann, M. D. Englen, P. J. FedorkaCray, and M. A. Harrison, "23S rRNA gene mutations contributing to macrolide resistance in Campylobacter jejuni and Campylobacter coli," Foodborne Pathogens and Disease, vol. 6, no. 1, pp. 91-98, 2009.

[36] D. E. Taylor and N. Chang, "In vitro susceptibilities of Campylobacter jejuni and Campylobacter coli to azithromycin and erythromycin," Antimicrobial Agents and Chemotherapy, vol. 35, no. 9, pp. 1917-1918, 1991.

[37] S. Zhao, G. H. Tyson, Y. Chen et al., "Whole-genome sequencing analysis accurately predicts antimicrobial resistance phenotypes in Campylobacter spp," Applied and Environmental Microbiology, vol. 82, no. 2, pp. 459-466, 2016.

[38] P. A. Olah, C. Doetkott, M. K. Fakhr, and C. M. Logue, "Prevalence of the Campylobacter multi-drug efflux pump (CmeABC) in Campylobacter spp. isolated from freshly processed turkeys," Food Microbiology, vol. 23, no. 5, pp. 453-460, 2006.

[39] C. Cagliero, L. Cloix, A. Cloeckaert, and S. Payot, "High genetic variation in the multidrug transporter cmeB gene in Campylobacter jejuni and Campylobacter coli," Journal of Antimicrobial Chemotherapy, vol. 58, no. 1, pp. 168-172, 2006.

[40] Y. Wang, M. Zhang, F. Deng et al., "Emergence of multidrugresistant Campylobacter species isolates with a horizontally acquired rRNA methylase," Antimicrobial Agents and Chemotherapy, vol. 58, no. 9, pp. 5405-5412, 2014.

[41] D. Liu, F. Deng, Y. Gao et al., "Dissemination of erm(B) and its associated multidrug-resistance genomic islands in Campylobacter from 2013 to 2015," Veterinary Microbiology, vol. 204, pp. 20-24, 2017.

[42] I. Kempf, A. Kerouanton, S. Bougeard et al., "Campylobacter coli in organic and conventional pig production in France and Sweden: Prevalence and antimicrobial resistance," Frontiers in Microbiology, vol. 8, 2017.

[43] C. U. Köser, M. J. Ellington, and S. J. Peacock, "Wholegenome sequencing to control antimicrobial resistance," Trends in Genetics, vol. 30, no. 9, pp. 401-407, 2014.

[44] M. Kurinčič, N. Botteldoorn, L. Herman, and S. Smole Možina, "Mechanisms of erythromycin resistance of Campylobacter spp. isolated from food, animals and humans," International Journal of Food Microbiology, vol. 120, no. 1-2, pp. 186-190, 2007.

[45] J. Lin, M. Yan, O. Sahin, S. Pereira, Y.-J. Chang, and Q. Zhang, "Effect of macrolide usage on emergence of erythromycinresistant Campylobacter isolates in chickens," Antimicrobial Agents and Chemotherapy, vol. 51, no. 5, pp. 1678-1686, 2007.

[46] D. B. Caldwell, Y. Wang, and J. Lin, "Development, stability, and molecular mechanisms of macrolide resistance in Campylobacter jejuni," Antimicrobial Agents and Chemotherapy, vol. 52, no. 11, pp. 3947-3954, 2008. 


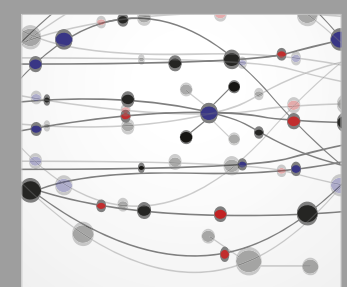

The Scientific World Journal
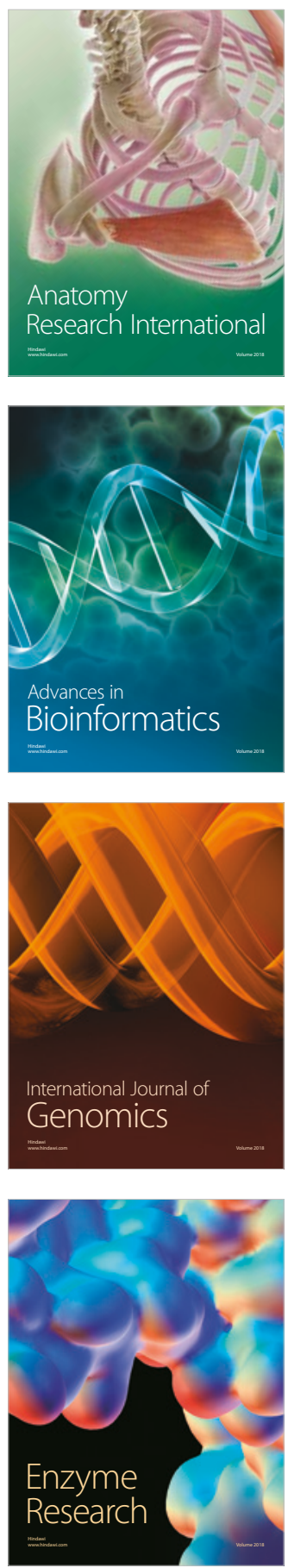
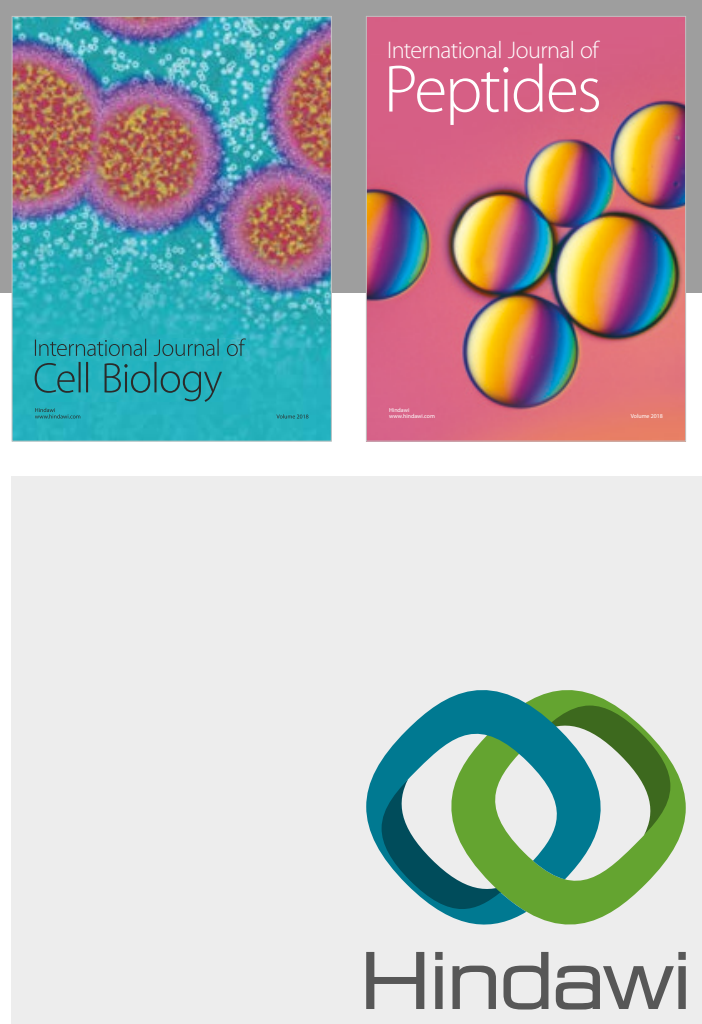

Submit your manuscripts at

www.hindawi.com
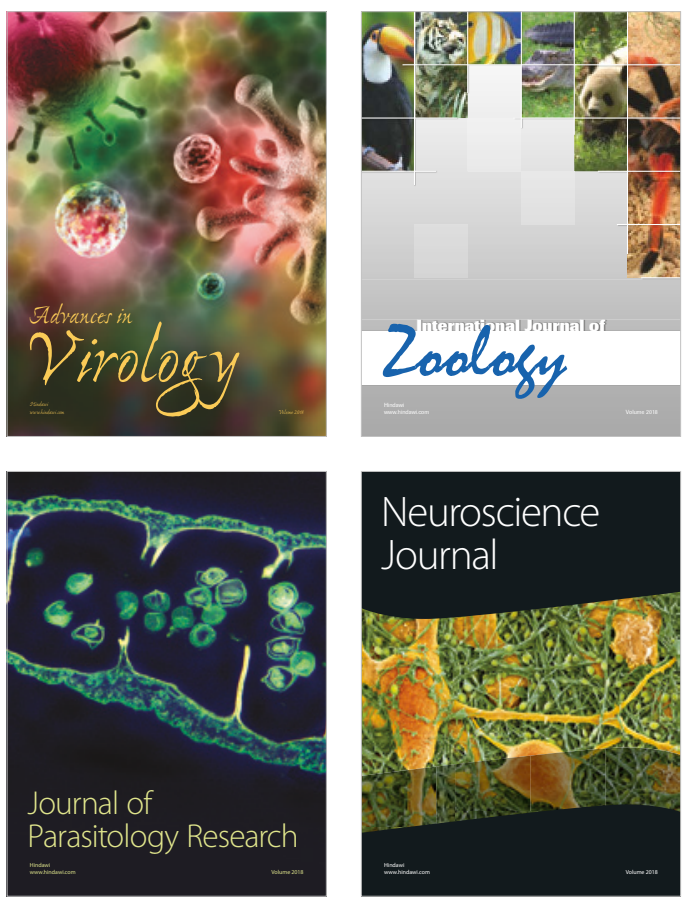
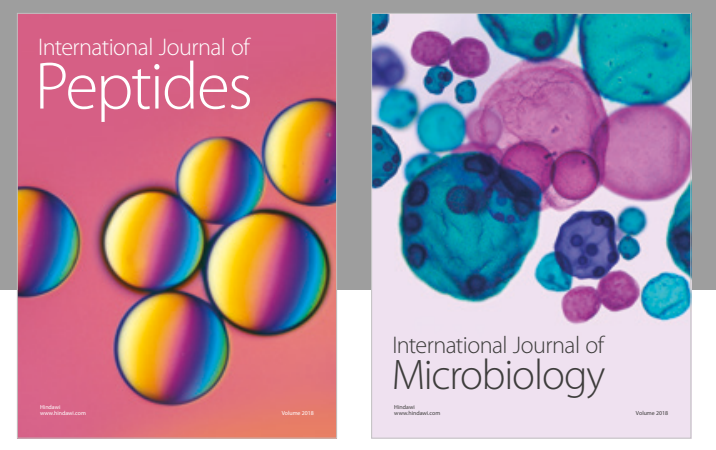

nternational Journal of Microbiology
Journal of
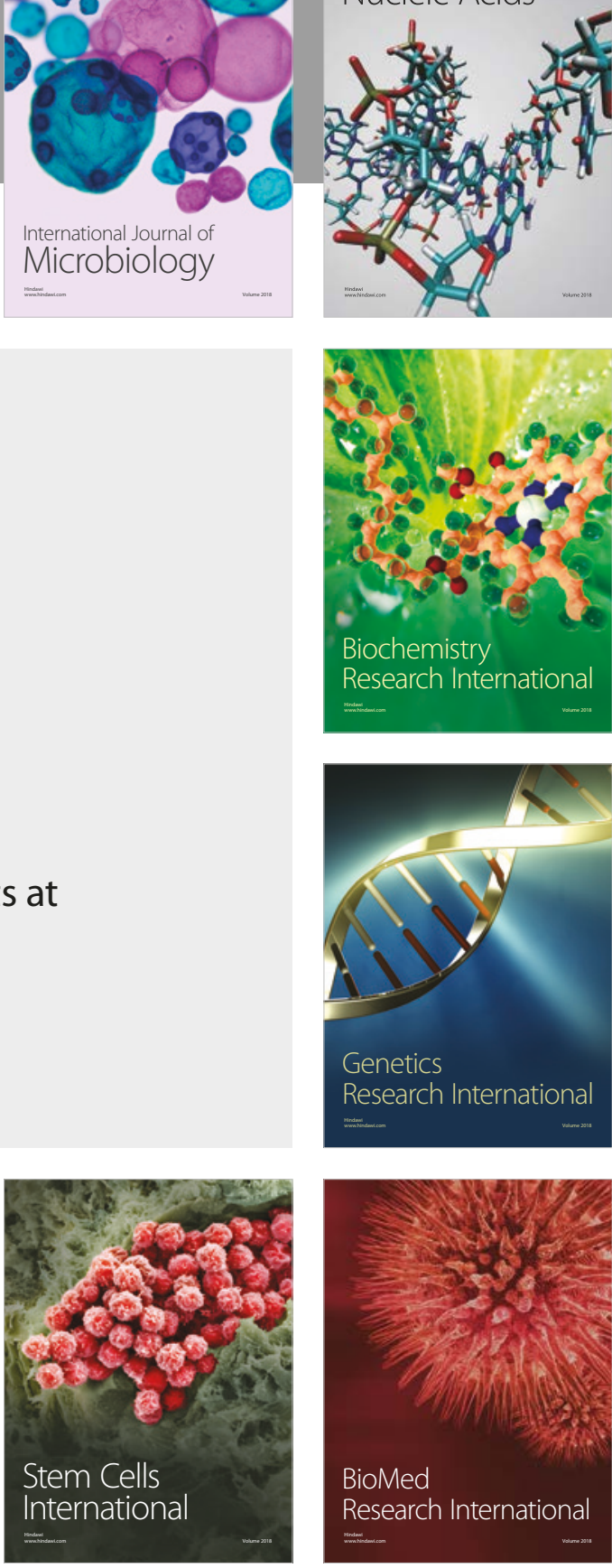
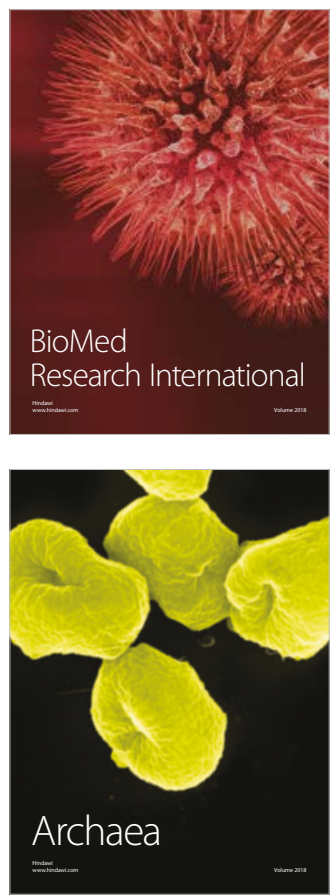